\title{
Existence of Countably Many Positive Solutions for Nonlinear Boundary Value Problems on Time Scales
}

\author{
Abdulkadir Dogan* \\ Department of Applied Mathematics, Faculty of Computer Sciences, Abdullah Gul University, Kayseri, 38039, Turkey
}

Received: 14 Aug. 2013, Revised: 11 Nov. 2013, Accepted: 12 Nov. 2013

Published online: 1 Sep. 2014

\begin{abstract}
In this paper, we consider the existence of countably many positive solutions for nonlinear singular boundary value problem on time scales. By using the fixed-point index theory and a new fixed-point theorem in cones, the sufficient conditions for the existence of countably many positive solutions are established.
\end{abstract}

Keywords: Time scales, Boundary value problem, Singularity, Positive solutions, Fixed point theorem

\section{Introduction}

The theory of time scales, which has recently received a lot of attention, was introduced and developed by Aulbach and Hilger [11] in 1988. It has been created in order to unify continuous and discrete analysis, and it allows a simultaneous treatment of differential and difference equations, extending those theories to so-called dynamic equations. Further, the study of time scales has led to several important applications, e.g., in the study of insect population models, heat transfer, neural networks, phytoremediation of metals, wound healing, and epidemic models.

In this paper, we are interested in the existence of countably many positive solutions for singular multipoint boundary value problem on time scales,

$$
\left(\phi\left(u^{\Delta}(t)\right)\right)^{\nabla}+a(t) f(u(t))=0, \quad t \in[0, T]_{\mathbb{T}},
$$

subject to following boundary condition

$$
u^{\Delta}(0)=0, \quad u(T)=\sum_{i=1}^{m-2} b_{i} u\left(\xi_{i}\right)
$$

where $\phi: R \longrightarrow R$ is an increasing homeomorphism and positive homomorphism and $\phi(0)=0 . \quad \xi_{i} \in[0, T]_{\mathbb{T}}$ with $0<\xi_{1}<\xi_{2}<\cdots<\xi_{m-2}<T$, and $b_{i}$ satisfies $b_{i} \in[0, T]_{\mathbb{T}}, \quad 0<\sum_{i=1}^{m-2} b_{i}<1, a(t):[0, T]_{\mathbb{T}} \rightarrow[0,+\infty]$ and has countably many singularities in $[0, T]_{\mathbb{T}}$. The usual notation and terminology for time scales as can be found in $[2,3]$, will be used here.
A projection $\phi: R \longrightarrow R$ is called an increasing homeomorphism and positive homomorphism if the following conditions are satisfied:

(i) if $x \leq y$, then $\phi(x) \leq \phi(y), \forall x, y \in R$;

(ii) $\phi$ is a continuous bijection and its inverse mapping is also continuous;

(iii) $\phi(x y)=\phi(x) \phi(y), \forall x, y \in R$.

If the above conditions hold, then it implies that $\phi$ is homogeneous and generates a $p$-Laplacian operator, i.e., $\phi(u)=|u|^{p-2} u$, for some $p>1$.

In recent years, there is much attention focused on on the existence of positive solutions of boundary value problems on time scales, some authors have found many results; for details, see $[1,5,6,7,8,9,10,13,19,20,21,22$, $23,24,25]$ and the references therein. But for the existence of countable many positive solutions for boundary value problem on time scales, few works were done as far as we know $[14,16]$.

We would like to mention the results of Ma et al. [17], Liang and Zhang [15] and Ji et al. [12].

Ma et al. [17] studied the existence of monotone positive solutions for the BVP

$$
\begin{aligned}
&\left(\phi_{p}\left(u^{\prime}\right)\right)^{\prime}+q(t) f(t, u)=0, \quad t \in(0,1), \\
& u^{\prime}(0)=\sum_{i=1}^{n} \alpha_{i} u^{\prime}\left(\xi_{i}\right), \quad u(1)=\sum_{i=1}^{n} \beta_{i} u\left(\xi_{i}\right),
\end{aligned}
$$

where $\xi_{i} \in(0,1)$ and $0 \leq \alpha_{i}, \beta_{i}<1$ satisfy $0 \leq \sum_{i=1}^{n} \alpha_{i}, \sum_{i=1}^{n} \beta_{i}<1$. The main tool is the monotone iterative technique.

\footnotetext{
*Corresponding author e-mail: abdulkadir.dogan@agu.edu.tr
} 
Liang and Zhang [15] considered the existence of countably many positive solutions for singular BVP

$$
\begin{aligned}
& \left.\left(\varphi_{p}\left(u^{\prime}\right)\right)^{\prime}+a(t) f(u(t))\right)=0, \quad t \in(0,1), \\
& u(0)=\sum_{i=1}^{m-2} \alpha_{i} u\left(\xi_{i}\right), \quad \varphi\left(u^{\prime}(1)\right)=\sum_{i=1}^{m-2} \beta_{i} \varphi\left(u^{\prime}\left(\xi_{i}\right)\right),
\end{aligned}
$$

where $\varphi: R \rightarrow R$ is a increasing homeomorphism and positive homomorphism and $\varphi(0)=0, \xi_{i} \in(0,1)$ with $0<\xi_{1}<\xi_{2}<\cdots<\xi_{m-2}<1$, $\alpha_{i}, \beta_{i} \in[0,+\infty), 0<\sum_{i=1}^{m-2} \alpha_{i}<1,0<\sum_{i=1}^{m-2} \beta_{i}<1$. They showed that there exist countably many positive solutions by using the fixed-point index theory and a new fixed-point theorem in cones.

Ji et al. [12] found the existence of countably many positive solutions for a singular multipoint BVP

$$
\begin{aligned}
\left(\phi_{p}\left(u^{\prime}\right)\right)^{\prime}(t)+a(t) f(u(t)) & =0, \quad t \in(0,1), \\
u^{\prime}(0)-\sum_{i=1}^{m-2} \alpha_{i} u\left(\xi_{i}\right) & =0, \quad u^{\prime}(1)+\sum_{i=1}^{m-2} \alpha_{i} u\left(\eta_{i}\right)=0,
\end{aligned}
$$

where $\phi_{p}(s)=|s|^{p-2} s, p>1,\left(\phi_{p}\right)^{-1}=\phi_{q}$ and $\frac{1}{p}+\frac{1}{q}=1$. They provided sufficient conditions for the existence of countably many positive solutions by using fixed-point index theory and the Leggett-Williams' fixed point theorem.

However, to the best of our knowledge, no work has been done for BVP (1) and (2). The aim of this paper is to fill the gap in the relevant literature.

Throughout the paper, we will suppose that the following conditions are satisfied:

(H1) $f:[0,+\infty) \rightarrow[0,+\infty)$ is continuous;

(H2) There exists a sequence $\left\{t_{i}\right\}_{i=1}^{\infty}$ such that $0<t_{i+1}<$ $t_{i}<\frac{T}{2}, \lim _{i \rightarrow \infty} t_{i}=t_{0}<\frac{T}{2}$, and $t_{0} \in[0, T]_{\mathbb{T}} . \lim _{t \rightarrow t_{i}}=$ $\infty, i=1,2, \ldots$, and

$$
0<\int_{0}^{T} a(s) \nabla s<+\infty .
$$

Moreover, $a(t)$ does not vanish identically on any subinterval of $[0, T]_{\mathbb{T}}$.

The rest of paper is arranged as follows. In Section 2, we state some definitions, notations, lemmas and prove several preliminary results. Section 3 is devoted to the presentation and proof of our main results. In last section 4 , we present an example of a family of functions $a(t)$ that satisfy condition $(\mathrm{H} 2)$.

\section{Preliminaries}

In this section, we provide some background materials from theory of cones in Banach spaces.

Definition 2.1. Let $E$ be a real Banach space. A nonempty, closed, convex set $P \subset E$ is a cone if it satisfies the following two conditions: (i) $x \in P, \quad \lambda \geq 0$ imply $\quad \lambda x \in P$;

(ii) $x \in P, \quad-x \in P$ imply $\quad x=0$.

Every cone $P \subset E$ induces an ordering in $E$ given by $x \leq y$ if and only if $y-x \in P$.

Definition 2.2. A map $\alpha$ is said to be a nonnegative continuous concave functional on a cone $P$ of a real Banach space $E$ if $\alpha: P \rightarrow[0, \infty)$ is continuous, and

$$
\alpha(t x+(1-t) y) \leq t \alpha(x)+(1-t) \alpha(y)
$$

for all $x, y \in P$ and $t \in[0,1]$.

Definition 2.3. Given a nonnegative continuous functional $\gamma$ on a cone $P$ of $E$, for each $d>0$ we define the set

$$
P(\gamma, d)=\{x \in P: \gamma(x)<d\} .
$$

The following fixed point theorems will play an important role in the proofs of our main results.

Theorem 2.1. ([4]). Let $E$ be a Banach space and $P \subset E$ be a cone in $E$. For $r>0$, define $\Omega_{r}=\{u \in P:\|u\|<$ $r\}$. Assume that $T: P \cap \overline{\Omega_{r}} \rightarrow P$ is a completely continuous operator such that $T u \neq u$ for $u \in \partial \Omega_{r}$;

(a) If $\|T u\| \leq\|u\|$ for $u \in \partial \Omega_{r}$, then $i\left(T, \Omega_{r}, P\right)=1$;

(b) If $\|T u\| \geq\|u\|$ for $u \in \partial \Omega_{r}$, then $i\left(T, \Omega_{r}, P\right)=0$.

Theorem 2.2. ([18]). Let $P$ be a cone in a Banach space $E$. Let $\alpha, \beta$ and $\gamma$ be three increasing, nonnegative and continuous functionals on $P$, satisfying for some $c>0$ and $M>0$ such that

$$
\gamma(u) \leq \beta(u) \leq \alpha(u), \quad\|u\| \leq M \gamma(u)
$$

for all $u \in \overline{P(\gamma, c)}$. Suppose there exists a completely continuous operator $T: \overline{P(\gamma, c)} \rightarrow P$ and $0<a<b<c$ such that

(S1) $\gamma(T u)<c$, for all $u \in \partial P(\gamma, c)$;

(S2) $\beta(T u)>b$, for all $u \in \partial P(\beta, b)$;

(S3) $P(\alpha, a) \neq \emptyset$, and $\alpha(T u)<a$, for all $u \in \partial P(\alpha, a)$.

Then $T$ has at least three fixed points $u_{1}, u_{2}, u_{3} \in \overline{P(\gamma, c)}$ such that

$0 \leq \alpha\left(u_{1}\right)<a<\alpha\left(u_{2}\right), \quad \beta\left(u_{2}\right)<b<\beta\left(u_{3}\right), \quad \gamma\left(u_{3}\right)<c$.

Lemma 2.1. If $\quad \sum_{i=1}^{m-2} b_{i} \neq 1$, then for $h \in C_{l d}[0, T]_{\mathbb{T}}$ and $h \geq 0$,

$$
\left.\phi\left(u^{\Delta}(t)\right)\right)^{\nabla}+h(t)=0, \quad t \in[0, T],
$$

$$
u^{\Delta}(0)=0, \quad u(T)=\sum_{i=1}^{m-2} b_{i} u\left(\xi_{i}\right)
$$

has the unique solution

$$
u(t)=\int_{0}^{t} \phi^{-1}\left(\int_{s}^{T} h(r) \nabla r+A\right) \Delta s+B,
$$


where

$$
\begin{gathered}
A=-\int_{0}^{T} h(r) \nabla r \\
B=\frac{1}{1-\sum_{i=1}^{m-2} b_{i}}\left[\sum_{i=1}^{m-2} b_{i} \int_{0}^{\xi_{i}} \phi^{-1}\left(\int_{s}^{T} h(r) \nabla r+A\right) \Delta s\right. \\
\left.-\int_{0}^{T} \phi^{-1}\left(\int_{s}^{T} h(r) \nabla r+A\right) \Delta s\right] .
\end{gathered}
$$

Proof. Let $u$ be as in (5), taking the delta derivative of (5), we have

$u^{\Delta}(t)=\phi^{-1}\left(\int_{t}^{T} h(r) \nabla r+A\right)$,

moreover, we get

$\phi\left(u^{\Delta}(t)\right)=\int_{t}^{T} h(r) \nabla r+A$

taking the nabla derivative of this expression yields

$\left(\phi\left(u^{\Delta}(t)\right)\right)^{\nabla}=-h(t)$.

Routine calculations verify that $u$ satisfies the boundary value conditions in (4), so that $u$ given in (5) is a solution of (3) and (4). It is easy to see that BVP $\left(\phi\left(u^{\Delta}\right)\right)^{\nabla}=0$, $u^{\Delta}(0)=0, u(T)=\sum_{i=1}^{m-2} b_{i} u\left(\xi_{i}\right)$ has only the trivial solution. Thus $u$ in (5) is the unique solution of (3) and (4). The proof is complete.

Lemma 2.2. The solution of BVP (3) and (4) satisfies $u(t) \geq 0$, for $t \in[0, T]_{\mathbb{T}}$.

Proof. Let

$$
\varphi_{0}(s)=\phi^{-1}\left(\int_{s}^{T} h(r) \nabla r-\int_{0}^{T} h(r) \nabla r\right)
$$

Since

$$
\int_{s}^{T} h(r) \nabla r \leq \int_{0}^{T} h(r) \nabla r
$$

it follows that $\varphi_{0}(s) \leq 0$. According to Lemma 2.1, we get

$$
\begin{aligned}
u(0) & =B \\
& =\frac{\sum_{i=1}^{m-2} b_{i} \int_{0}^{\xi_{i}} \varphi_{0}(s) \Delta s-\int_{0}^{T} \varphi_{0}(s) \Delta s}{1-\sum_{i=1}^{m-2} b_{i}} \\
& =\frac{\sum_{i=1}^{m-2} b_{i}\left(\int_{0}^{T} \varphi_{0}(s) \Delta s-\int_{\xi_{i}}^{T} \varphi_{0}(s) \Delta s\right)-\int_{0}^{T} \varphi_{0}(s) \Delta s}{1-\sum_{i=1}^{m-2} b_{i}} \\
& =-\left[\int_{0}^{T} \varphi_{0}(s) \Delta s+\frac{\sum_{i=1}^{m-2} b_{i} \int_{\xi_{i}}^{T} \varphi_{0}(s) \Delta s}{1-\sum_{i=1}^{m-2} b_{i}}\right] \geq 0
\end{aligned}
$$

and

$$
\begin{aligned}
u(T)= & \int_{0}^{T} \varphi_{0}(s) \Delta s+\frac{\sum_{i=1}^{m-2} b_{i} \int_{0}^{\xi_{i}} \varphi_{0}(s) \Delta s-\int_{0}^{T} \varphi_{0}(s) \Delta s}{1-\sum_{i=1}^{m-2} b_{i}} \\
= & \frac{-\sum_{i=1}^{m-2} b_{i} \int_{0}^{T} \varphi_{0}(s) \Delta s+\sum_{i=1}^{m-2} b_{i} \int_{0}^{\xi_{i}} \varphi_{0}(s) \Delta s}{1-\sum_{i=1}^{m-2} b_{i}} \\
= & \frac{-\left[\sum_{i=1}^{m-2} b_{i}\left(\int_{0}^{T} \varphi_{0}(s) \Delta s-\int_{0}^{\xi_{i}} \varphi_{0}(s) \Delta s\right)\right]}{1-\sum_{i=1}^{m-2} b_{i}} \\
= & \frac{-\sum_{i=1}^{m-2} b_{i} \int_{\xi_{i}}^{T} \varphi_{0}(s) \Delta s}{1-\sum_{i=1}^{m-2} b_{i}} \geq 0 .
\end{aligned}
$$

If $t \in(0, T)$, we have

$$
\begin{aligned}
u(t)= & \int_{0}^{t} \varphi_{0}(s) \Delta s+\frac{1}{1-\sum_{i=1}^{m-2} b_{i}}\left[\sum_{i=1}^{m-2} b_{i} \int_{0}^{\xi_{i}} \varphi_{0}(s) \Delta s\right. \\
& \left.-\int_{0}^{T} \varphi_{0}(s) \Delta s\right] \\
= & \frac{1}{1-\sum_{i=1}^{m-2} b_{i}}\left[\int_{0}^{t} \varphi_{0}(s) \Delta s-\sum_{i=1}^{m-2} b_{i} \int_{0}^{t} \varphi_{0}(s) \Delta s\right. \\
& \left.+\sum_{i=1}^{m-2} b_{i} \int_{0}^{\xi_{i}} \varphi_{0}(s) \Delta s-\int_{0}^{T} \varphi_{0}(s) \Delta s\right] \\
\geq & \frac{1}{1-\sum_{i=1}^{m-2} b_{i}}\left[\int_{0}^{T} \varphi_{0}(s) \Delta s-\sum_{i=1}^{m-2} b_{i} \int_{0}^{T} \varphi_{0}(s) \Delta s\right. \\
& \left.+\sum_{i=1}^{m-2} b_{i} \int_{0}^{\xi_{i}} \varphi_{0}(s) \Delta s-\int_{0}^{T} \varphi_{0}(s) \Delta s\right] \\
= & \frac{-\sum_{i=1}^{m-2} b_{i}\left(\int_{0}^{T} \varphi_{0}(s) \Delta s-\int_{0}^{\xi_{i}} \varphi_{0}(s) \Delta s\right)}{1-\sum_{i=1}^{m-2} b_{i}} \\
= & \frac{-\sum_{i=1}^{m-2} b_{i} \int_{\xi_{i}}^{T} \varphi_{0}(s) \Delta s}{1-\sum_{i=1}^{m-2} b_{i}} \geq 0 .
\end{aligned}
$$

So $u(t) \geq 0, \quad t \in[0, T]$. The proof is complete.

Lemma 2.3. If $u \in P$, then

$u(t) \geq \frac{\theta}{T}\|u\|, \quad t \in[\theta, T-\theta]$,

where

$\|u\|=\sup _{t \in[0, T]_{\mathbb{T}}}|u(t)|$.

Proof. Let

$$
\tau=\inf \left\{\xi \in[0, T]: \sup _{t \in[0, T]_{\mathbb{T}}} u(t)=u(\xi)\right\} .
$$

Case (i). $\tau \in[0, \theta]$. It follows from the concavity of $u(t)$ that each point on chord between $(\tau, u(\tau))$ and $(T, u(T))$ 
is below the graph of $u(t)$. Thus,

$$
u(t) \geq u(\tau)+\frac{u(T)-u(\tau)}{T-\tau}(t-\tau), \quad t \in[\theta, T-\theta] .
$$

Hence,

$$
\begin{aligned}
u(t) & \geq \min _{t \in[\theta, T-\theta]}\left[u(\tau)+\frac{u(T)-u(\tau)}{T-\tau}(t-\tau)\right] \\
& =u(\tau)+\frac{u(T)-u(\tau)}{T-\tau}(T-\theta-\tau) \\
& =\frac{T-\theta-\tau}{T-\tau} u(T)+\frac{\theta}{T-\tau} u(\tau) \geq \frac{\theta}{T} u(\tau),
\end{aligned}
$$

which implies that

$$
u(t) \geq \frac{\theta}{T}\|u\|
$$

Case (ii). $\tau \in[\theta, T-\theta]$. If $t \in[\theta, \tau]$, similarly, we have

$$
u(t) \geq u(\tau)+\frac{u(\tau)-u(0)}{\tau}(t-\tau), \quad t \in[\theta, \tau] .
$$

Thus,

$$
\begin{aligned}
u(t) & \geq \min _{t \in[\theta, \tau]}\left[u(\tau)+\frac{u(\tau)-u(0)}{\tau}(t-\tau)\right] \\
& =u(\tau)+\frac{u(\tau)-u(0)}{\tau}(\theta-\tau) \\
& =\frac{\theta}{\tau} u(\tau)+\left(1-\frac{\theta}{\tau}\right) u(0) \geq \frac{\theta}{T} u(\tau) .
\end{aligned}
$$

If $t \in[\tau, T-\theta]$, similarly,

$$
u(t) \geq u(\tau)+\frac{u(T)-u(\tau)}{T-\tau}(t-\tau), \quad t \in[\tau, T-\theta] .
$$

Thus,

$$
\begin{aligned}
u(t) & \geq \min _{t \in[\tau, T-\theta]}\left[u(\tau)+\frac{u(T)-u(\tau)}{T-\tau}(t-\tau)\right] \\
& =u(\tau)+\frac{u(T)-u(\tau)}{T-\tau}(T-\theta-\tau) \\
& =\frac{\theta}{T-\tau} u(\tau)+\frac{T-\theta-\tau}{T-\tau} u(T) \geq \frac{\theta}{T} u(\tau) .
\end{aligned}
$$

Therefore, we find

$$
u(t) \geq \frac{\theta}{T}\|u\|, \quad t \in[\theta, T-\theta]
$$

Case (iii). $\tau \in[T-\theta, T]$. Similarly, we have

$$
u(t) \geq u(\tau)+\frac{u(T)-u(0)}{\tau}(t-\tau), \quad t \in[\theta, T-\theta] .
$$

Thus,

$$
\begin{aligned}
u(t) & \geq \min _{t \in[\theta, T-\theta]}\left[u(\tau)+\frac{u(\tau)-u(0)}{\tau}(t-\tau)\right] \\
& =u(\tau)+\frac{u(\tau)-u(0)}{\tau}(\theta-\tau) \\
& =\frac{\theta}{\tau} u(\tau)+\left(1-\frac{\theta}{\tau}\right) u(0) \geq \frac{\theta}{T} u(\tau)
\end{aligned}
$$

which yields

$$
u(t) \geq \frac{\theta}{T}\|u\|, \quad t \in[\theta, T-\theta] .
$$

This completes the proof.

\section{Existence of positive solutions}

Let the Banach space $E=C_{l d}\left([0, T]_{\mathbb{T}}, \mathbb{R}\right)$ with norm $\|u\|=$ $\sup _{[0, T]_{\mathbb{T}}}|u(t)|$ and define the cone $P \subset E$ by

$P=\{u \in E: u(t)$ is a nondecreasing concave and nonnegative function on $\left.[0, T]_{\mathbb{T}}\right\}$.

Let $\theta_{k}<r_{k}<T-\theta_{k}$, we define the nonnegative, increasing, continuous functional $\gamma_{k}, \beta_{k}$, and $\alpha_{k}$ by

$$
\begin{aligned}
& \gamma_{k}(u)=\max _{t \in\left[\theta_{k}, r_{k}\right]_{\mathbb{T}}} u(t)=u\left(r_{k}\right), \\
& \beta_{k}(u)=\min _{t \in\left[r_{k}, T-\theta_{k}\right]_{\mathbb{T}}} u(t)=u\left(r_{k}\right), \\
& \alpha_{k}(u)=\max _{t \in\left[\theta_{k}, T-\theta_{k}\right]_{\mathbb{T}}} u(t)=u\left(T-\theta_{k}\right) .
\end{aligned}
$$

It is easy to see that for each $u \in P$,

$\gamma_{k}(u) \leq \beta_{k}(u) \leq \alpha_{k}(u)$

Moreover, by Lemma 2.3, for each $u \in P$,

$\gamma_{k}(u)=u\left(r_{k}\right) \geq \frac{\theta_{k}}{T}\|u\|$

Define the operator $T: P \longrightarrow E$ by

$$
\begin{aligned}
(T u)(t)= & \int_{0}^{t} \phi^{-1}\left(\int_{s}^{T} a(r) f(u(r)) \nabla r\right. \\
& \left.-\int_{0}^{T} a(r) f(u(r)) \nabla r\right) \Delta s \\
& +\frac{\sum_{i=1}^{m-2} b_{i} \int_{0}^{\xi_{i}} \phi^{-1}\left(\int_{s}^{T} a(r) f(u(r)) \nabla r\right.}{1-\sum_{i=1}^{m-2} b_{i}} \\
& -\frac{\left.\int_{0}^{T} a(r) f(u(r)) \nabla r\right) \Delta s}{1-\sum_{i=1}^{m-2} b_{i}} \\
& -\frac{\int_{0}^{T} \phi^{-1}\left(\int_{s}^{T} a(r) f(u(r)) \nabla r\right.}{1-\sum_{i=1}^{m-2} b_{i}} \\
& -\frac{\left.\int_{0}^{T} a(r) f(u(r)) \nabla r\right) \Delta s}{1-\sum_{i=1}^{m-2} b_{i}} .
\end{aligned}
$$

It is easy to see that $(T u)(t) \geq 0, \quad(T u)^{\Delta}(0)=$ $0, \quad(T u)(T)=\sum_{i=1}^{m-2} b_{i}(T u)\left(\xi_{i}\right) \quad$ and 
$\left[\phi(T u)^{\Delta}(t)\right]^{\nabla}=-a(t) f(u(t)) \leq 0$. This shows $T P \subset P$. One may show that $T: P \rightarrow P$ is completely continuous.

Lemma 3.1. Assume condition $(\mathrm{H} 2)$ holds. Then there exists a constant $\theta \in \max \left\{t \in T: 0<t<\frac{T}{2}\right\}$ satisfies

$0<\int_{\theta}^{T-\theta} a(s) \nabla s<+\infty$

Furthermore, the function

$$
\begin{aligned}
A(t)= & \int_{t}^{T-t_{1}} \phi^{-1}\left(\int_{s}^{T-t_{1}} a(r) \nabla r-\int_{0}^{T-t_{1}} a(r) \nabla r\right) \Delta s \\
& +\frac{\sum_{i=1}^{m-2} b_{i} \int_{t_{1}}^{t} \phi^{-1}\left(\int_{s}^{t} a(r) \nabla r-\int_{0}^{t} a(r) \nabla r\right) \Delta s}{1-\sum_{i=1}^{m-2} b_{i}} \\
& -\frac{\int_{t_{1}}^{t} \phi^{-1}\left(\int_{s}^{t} a(r) \nabla r-\int_{0}^{t} a(r) \nabla r\right) \Delta s}{1-\sum_{i=1}^{m-2} b_{i}}
\end{aligned}
$$

is a positive continuous function on $\left[t_{1}, T-t_{1}\right]$ and has a minimum on $\left[t_{1}, T-t_{1}\right]$, therefore there exist $L>0$ such that $A(t) \geq 0, t \in\left[t_{1}, T-t_{1}\right]$.

Proof. At first, it is easily seen that $A(t)$ is continuous on $\left[t_{1}, T-t_{1}\right]$. Next, let

$$
\begin{aligned}
A_{1}(t)= & \int_{t}^{T-t_{1}} \phi^{-1}\left(\int_{s}^{T-t_{1}} a(r) \nabla r-\int_{0}^{T-t_{1}} a(r) \nabla r\right) \Delta s \\
A_{2}(t)= & \frac{\sum_{i=1}^{m-2} b_{i} \int_{t_{1}}^{t} \phi^{-1}\left(\int_{s}^{t} a(r) \nabla r-\int_{0}^{t} a(r) \nabla r\right) \Delta s}{1-\sum_{i=1}^{m-2} b_{i}} \\
& -\frac{\int_{t_{1}}^{t} \phi^{-1}\left(\int_{s}^{t} a(r) \nabla r-\int_{0}^{t} a(r) \nabla r\right) \Delta s}{1-\sum_{i=1}^{m-2} b_{i}}
\end{aligned}
$$

Then, from condition ( $\mathrm{H} 2)$, we have the function $A_{1}(t)$ is strictly monotone decreasing on $\left[t_{1}, T-t_{1}\right]$ and $A_{1}\left(T-t_{1}\right)=0$, the function $A_{2}(t)$ is strictly monotone increasing on $\left[t_{1}, T-t_{1}\right]$ and $A_{2}\left(t_{1}\right)=0$. Because $A_{1}(t)$ and $A_{2}(t)$ are not equal to zero at the same time. Therefore the function $A(t)=A_{1}(t)+A_{2}(t)$ is positive on $\left[t_{1}, T-t_{1}\right]$, which implies $L=\min _{t \in\left[t_{1}, T-t_{1}\right]} A(t)>0$. The proof is complete.

For convenience, we denote by

$\lambda_{1}=1 / L$,

$$
\begin{aligned}
\lambda_{2}= & 1 /\left\{\int_{0}^{T} \phi^{-1}\left(\int_{0}^{T} a(r) \nabla r\right) \Delta s\right. \\
& +\frac{1}{1-\sum_{i=1}^{m-2} b_{i}}\left[\sum_{i=1}^{m-2} b_{i} \int_{0}^{\xi_{i}} \phi^{-1}\left(\int_{0}^{T} a(r) \nabla r\right) \Delta s\right. \\
& \left.\left.-\int_{0}^{T} \phi^{-1}\left(\int_{s}^{T} a(r) \nabla r\right) \Delta s\right]\right\} .
\end{aligned}
$$

Theorem 3.1. Assume that $(\mathrm{H} 1)$ and $(\mathrm{H} 2)$ hold. Let $\left\{\theta_{k}\right\}_{k=1}^{\infty}$ be such that $\theta_{k} \in\left(t_{k+1}, t_{k}\right),(k=1,2, \ldots)$. Let $\left\{r_{k}\right\}_{k=1}^{\infty}$ and $\left\{R_{k}\right\}_{k=1}^{\infty}$ be such that

$R_{k+1}<\frac{\theta_{k}}{T} r_{k}<r_{k}<m r_{k}<R_{k}, m r_{k} \leq M R_{k}, \quad k=1,2, \ldots$
Furthermore for each natural number $k$, we assume that $f$ satisfies:

(H3) $f(u) \geq \phi\left(m r_{k}\right)$, for all $u \in\left[\frac{\theta_{k}}{T} r_{k}, r_{k}\right]$,

(H4) $f(u) \leq \phi\left(M R_{k}\right)$, for all $u \in\left[0, R_{k}\right]$,

where $m \in\left(\lambda_{1},+\infty\right), \quad M \in\left(0, \lambda_{2}\right)$. Then BVP (1) and (2) has infinitely many solutions $\left\{u_{k}\right\}_{k=1}^{\infty}$ such that

$$
r_{k} \leq\left\|u_{k}\right\| \leq R_{k}, \quad k=1,2, \ldots
$$

Proof. Because $0<t_{0} \leq t_{k+1}<\theta_{k}<t_{k}<T, \quad k=1,2, \ldots$, then for any $k \in N, u \in P$, by Lemma 2.3, we get

$$
u(t) \geq \frac{\theta_{k}}{T}\|u\|, \quad t \in\left[\theta_{k}, T-\theta_{k}\right]
$$

We consider the sequence $\left\{\Omega_{1, k}\right\}_{k=1}^{\infty}$ and $\left\{\Omega_{2, k}\right\}_{k=1}^{\infty}$ of open subsets of $E$ defined by

$$
\Omega_{1, k}=\left\{u \in P:\|u\|<r_{k}\right\}, \quad k=1,2, \ldots,
$$

$$
\Omega_{2, k}=\left\{u \in P:\|u\|<R_{k}\right\}, \quad k=1,2, \ldots
$$

For a fixed $k$ and $u \in \partial \Omega_{1, k}$, by (6) we have

$$
\begin{aligned}
r_{k} & =\|u\|=\sup _{0 \leq t \leq T}|u(t)| \\
& \geq \sup _{\theta_{k} \leq t \leq T-\theta_{k}} u(t) \geq \frac{\theta_{k}}{T}\|u\| \\
& =\frac{\theta_{k}}{T} r_{k}, \text { for all } t \in\left[\theta_{k}, T-\theta_{k}\right] .
\end{aligned}
$$

By condition (H3), we have

$$
f(u) \geq \phi\left(m r_{k}\right), \text { for all } t \in\left[\theta_{k}, T-\theta_{k}\right]
$$

Since $\left(t_{1}, T-t_{1}\right) \subset\left[\theta_{k}, T-\theta_{k}\right]$, if (H2) holds, we consider three possibilities:

(i) If $\xi_{1} \in\left[t_{1}, T-t_{1}\right], \quad$ then for $u \in \partial \Omega_{1, k}$, by (H3) and 


$$
\begin{aligned}
& \text { Lemma 3.1, we have } \\
& \begin{aligned}
&\|T u\|=\int_{0}^{T} \phi^{-1}\left(\int_{s}^{T} a(r) f(u(r)) \nabla r\right.-\frac{\left.\int_{0}^{\xi_{1}} a(r) \nabla r\right) \Delta s}{1-\sum_{i=1}^{m-2} b_{i}} \\
&\left.-\int_{0}^{T} a(r) f(u(r)) \nabla r\right) \Delta s\left.-\frac{\int_{t_{1}}^{\xi_{1}} \phi^{-1}\left(\int_{s}^{\xi_{1}} a(r) \nabla r-\int_{0}^{\xi_{1}} a(r) \nabla r\right) \Delta s}{1-\sum_{i=1}^{m-2} b_{i}}\right] \\
&
\end{aligned}
\end{aligned}
$$$$
\frac{\sum_{i=1}^{m-2} b_{i} \int_{0}^{\xi_{i}} \phi^{-1}\left(\int_{s}^{T} a(r) f(u(r)) \nabla r\right.}{1-\sum_{i=1}^{m-2} b_{i}}
$$$$
-\frac{\left.\int_{0}^{T} a(r) f(u(r)) \nabla r\right) \Delta s}{1-\sum_{i=1}^{m-2} b_{i}}
$$$$
-\frac{\int_{0}^{T} \phi^{-1}\left(\int_{s}^{T} a(r) f(u(r)) \nabla r\right.}{1-\sum_{i=1}^{m-2} b_{i}}
$$$$
-\frac{\left.\int_{0}^{T} a(r) f(u(r)) \nabla r\right) \Delta s}{1-\sum^{m-2} b_{i}}
$$$$
\geq \int_{\xi_{1}}^{T-t_{1}} \phi^{-1}\left(\int_{s}^{T-t_{1}} a(r) f(u(r)) \nabla r\right.
$$$$
\left.-\int_{0}^{T-t_{1}} a(r) f(u(r)) \nabla r\right) \Delta s
$$$$
+\frac{\sum_{i=1}^{m-2} b_{i} \int_{t_{1}}^{t} \phi^{-1}\left(\int_{s}^{t} a(r) f(u(r)) \nabla r\right.}{1-\sum_{i=1}^{m-2} b_{i}}
$$$$
-\frac{\left.\int_{0}^{t} a(r) f(u(r)) \nabla r\right) \Delta s}{1-\sum_{i=1}^{m-2} b_{i}}
$$$$
-\frac{\int_{t_{1}}^{t} \phi^{-1}\left(\int_{s}^{t} a(r) f(u(r)) \nabla r\right.}{1-\sum_{i=1}^{m-2} b_{i}}
$$$$
-\frac{\left.\int_{0}^{t} a(r) f(u(r)) \nabla r\right) \Delta s}{1-\sum_{i=1}^{m-2} b_{i}}
$$$$
\geq\left(m r_{k}\right)\left[\int _ { \xi _ { 1 } } ^ { T - t _ { 1 } } \phi ^ { - 1 } \left(\int_{s}^{T-t_{1}} a(r) \nabla r\right.\right.
$$$$
\left.-\int_{0}^{T-t_{1}} a(r) \nabla r\right) \Delta s
$$$$
+\frac{\sum_{i=1}^{m-2} b_{i} \int_{t_{1}}^{\xi_{1}} \phi^{-1}\left(\int_{s}^{\xi_{1}} a(r) \nabla r\right.}{1-\sum_{i=1}^{m-2} b_{i}}
$$

(ii) If $\xi_{1} \in\left(T-t_{1}, T\right)$, then for $u \in \partial \Omega_{1, k}$, by (H3) and Lemma 3.1, we have

$$
\begin{gathered}
\|T u\| \geq \frac{\sum_{i=1}^{m-2} b_{i} \int_{t_{1}}^{T-t_{1}} \phi^{-1}\left(\int_{s}^{T-t_{1}} a(r) f(u(r)) \nabla r\right.}{1-\sum_{i=1}^{m-2} b_{i}} \\
-\frac{\left.\int_{0}^{T-t_{1}} a(r) f(u(r)) \nabla r\right) \Delta s}{1-\sum_{i=1}^{m-2} b_{i}} \\
\quad-\frac{\int_{t_{1}}^{T-t_{1}} \phi^{-1}\left(\int_{s}^{T-t_{1}} a(r) f(u(r)) \nabla r\right.}{1-\sum_{i=1}^{m-2} b_{i}} \\
\quad-\frac{\left.\int_{0}^{T-t_{1}} a(r) f(u(r)) \nabla r\right) \Delta s}{1-\sum_{i=1}^{m-2} b_{i}} \\
\geq m r_{k} A\left(T-t_{1}\right)>m r_{k} L>r_{k}=\|u\| .
\end{gathered}
$$

(iii) If $\xi_{1} \in\left(0, t_{1}\right)$, then for $u \in \partial \Omega_{1, k}$, by (H3) and Lemma 3.1, we have

$$
\begin{aligned}
\|T u\| \geq & \int_{0}^{T} \phi^{-1}\left(\int_{s}^{T} a(r) f(u(r)) \nabla r\right. \\
& \left.-\int_{0}^{T} a(r) f(u(r)) \nabla r\right) \Delta s \\
\geq & \int_{t_{1}}^{T-t_{1}} \phi^{-1}\left(\int_{s}^{T-t_{1}} a(r) f(u(r)) \nabla r\right. \\
& \left.-\int_{0}^{T-t_{1}} a(r) f(u(r)) \nabla r\right) \Delta s \\
\geq & m r_{k} \int_{t_{1}}^{T-t_{1}} \phi^{-1}\left(\int_{s}^{T-t_{1}} a(r) \nabla r\right. \\
& \left.-\int_{0}^{T-t_{1}} a(r) \nabla r\right) \Delta s \\
= & m r_{k} A\left(t_{1}\right) \geq m r_{k} L>r_{k}=\|u\| .
\end{aligned}
$$

Thus, in all cases, an application of Theorem 2.1 implies that

$$
i\left(T, \Omega_{1, k}, P\right)=0 \text {. }
$$

On the other hand, let $u \in \partial \Omega_{2, k}, \quad$ we have $u(t) \leq\|u\|=$ $R_{k}$, by (H4) we have

$$
f(u(t)) \leq \phi\left(M R_{k}\right), \text { for all } t \in[0, T]
$$


Therefore

$$
\begin{aligned}
& \|T u\|=\int_{0}^{T} \phi^{-1}\left(\int_{s}^{T} a(r) f(u(r)) \nabla r\right. \\
& \left.-\int_{0}^{T} a(r) f(u(r)) \nabla r\right) \Delta s \\
& +\frac{\sum_{i=1}^{m-2} b_{i} \int_{0}^{\xi_{i}} \phi^{-1}\left(\int_{s}^{T} a(r) f(u(r)) \nabla r\right.}{1-\sum_{i=1}^{m-2} b_{i}} \\
& -\frac{\left.\int_{0}^{T} a(r) f(u(r)) \nabla r\right) \Delta s}{1-\sum_{i=1}^{m-2} b_{i}} \\
& -\frac{\int_{0}^{T} \phi^{-1}\left(\int_{s}^{T} a(r) f(u(r)) \nabla r\right.}{1-\sum_{i=1}^{m-2} b_{i}} \\
& -\frac{\left.\int_{0}^{T} a(r) f(u(r)) \nabla r\right) \Delta s}{1-\sum_{i=1}^{m-2} b_{i}} \\
& \leq \int_{0}^{T} \phi^{-1}\left(\int_{0}^{T} a(r) f(u(r)) \nabla r\right) \Delta s \\
& +\frac{\sum_{i=1}^{m-2} b_{i} \int_{0}^{\xi_{i}} \phi^{-1}\left(\int_{0}^{T} a(r) f(u(r)) \nabla r\right) \Delta s}{1-\sum_{i=1}^{m-2} b_{i}} \\
& -\frac{\int_{0}^{T} \phi^{-1}\left(\int_{s}^{T} a(r) f(u(r)) \nabla r\right) \Delta s}{1-\sum_{i=1}^{m-2} b_{i}} \\
& \leq M R_{k}\left[\int_{0}^{T} \phi^{-1}\left(\int_{0}^{T} a(r) \nabla r\right) \Delta s\right. \\
& +\frac{\sum_{i=1}^{m-2} b_{i} \int_{0}^{\xi_{i}} \phi^{-1}\left(\int_{0}^{T} a(r) \nabla r\right) \Delta s}{1-\sum_{i=1}^{m-2} b_{i}} \\
& \left.-\frac{\int_{0}^{T} \phi^{-1}\left(\int_{s}^{T} a(r) \nabla r\right) \Delta s}{1-\sum_{i=1}^{m-2} b_{i}}\right]
\end{aligned}
$$$$
\leq R_{k}=\|u\| .
$$

Hence Theorem 2.1 implies that

$$
i\left(T, \Omega_{2, k}, P\right)=1 \text {. }
$$

Because $r_{k}<R_{k}$ for $k \in N$, (7) and (8), it follows from the additivity of the fixed point index that

$$
i\left(T, \Omega_{2, k} \backslash \bar{\Omega}_{1, k}, P\right)=1 \text {, for } k \in N .
$$

Thus, $T$ has a fixed point in $\Omega_{2, k} \backslash \bar{\Omega}_{1, k}$ such that $r_{k} \leq\left\|u_{k}\right\| \leq R_{k}$. Since $k \in N$ was arbitrary, the proof is completed.
For notational convenience, we denote $\rho_{k}$ and $\eta_{k}$ by

$$
\begin{aligned}
& \rho_{k}= \int_{0}^{T} \phi^{-1}\left(\int_{0}^{T} a(r) \nabla r\right) \Delta s \\
&+\frac{\sum_{i=1}^{m-2} b_{i} \int_{0}^{\xi_{i}} \phi^{-1}\left(\int_{0}^{T} a(r) \nabla r\right) \Delta s}{1-\sum_{i=1}^{m-2} b_{i}} \\
&-\frac{\int_{0}^{T} \phi^{-1}\left(\int_{s}^{T} a(r) \nabla r\right) \Delta s}{1-\sum_{i=1}^{m-2} b_{i}}, \\
& \eta_{k}=\int_{\theta_{k}}^{r_{k}} \phi^{-1}\left(\int_{s}^{T-\theta_{k}} a(r) \nabla r-\int_{0}^{T-\theta_{k}} a(r) \nabla r\right) \Delta s .
\end{aligned}
$$

Theorem 3.2. Suppose that (H1) and (H2) hold, and let $\left\{\theta_{k}\right\}_{k=1}^{\infty}$ be such that $\theta_{k} \in\left(t_{k+1}, t_{k}\right),(k=1,2, \ldots)$. Let $\left\{a_{k}\right\}_{k=1}^{\infty}, \quad\left\{b_{k}\right\}_{k=1}^{\infty}$ and $\left\{c_{k}\right\}_{k=1}^{\infty}$ be such that

$$
c_{k+1}<a_{k}<\frac{\theta_{k}}{T} b_{k}<b_{k}<c_{k},
$$

and

$$
\rho_{k} b_{k}<\eta_{k} c_{k}, \text { for } k=1,2, \ldots
$$

Furthermore for each natural number $k$ we assume that $f$ satisfies:

(H5) $f(u)<\phi\left(\frac{c_{k}}{\rho_{k}}\right)$, for all $0 \leq u(t) \leq \frac{T}{\theta_{k}} c_{k}$;

(H6) $f(u)>\phi\left(\frac{b_{k}}{\eta_{k}}\right)$, for all $b_{k} \leq u(t) \leq \frac{T}{\theta_{k}} b_{k}$;

(H7) $f(u)<\phi\left(\frac{a_{k}}{\rho_{k}}\right)$, for all $0 \leq u(t) \leq \frac{T}{\theta_{k}} a_{k}$.

Then the BVP (1) and (2) has three infinite families of solutions $\left\{u_{1 k}\right\}_{k=1}^{\infty}, \quad\left\{u_{2 k}\right\}_{k=1}^{\infty}$ and $\left\{u_{3 k}\right\}_{k=1}^{\infty}$ satisfying

$$
\begin{gathered}
0 \leq \alpha_{k}\left(u_{1 k}\right)<a_{k}<\alpha_{k}\left(u_{2 k}\right), \\
\beta_{k}\left(u_{2 k}\right)<b_{k}<\beta_{k}\left(u_{3 k}\right), \\
\gamma\left(u_{3 k}\right)<c_{k}, \text { for } k \in N .
\end{gathered}
$$

Proof. By the definition of the completely continuous operator $T$, it is easy to check that $T: \overline{P\left(\gamma_{k}, c_{k}\right)} \rightarrow P$, for $k \in N$.

We prove that all the conditions of Theorem 2.2 are satisfied. In order to make use of property (i) of Theorem 2.2, we chose $u \in \partial P\left(\gamma_{k}, c_{k}\right)$. Then $\gamma_{k}(u)=\max _{t \in\left[\theta_{k}, r_{k}\right]_{\mathbb{T}}} u(t)=u\left(r_{k}\right)=c_{k}$, this implies $0 \leq u(t) \leq c_{k} \quad$ for $t \in\left[0, r_{k}\right]_{\mathbb{T}}$. If we recall that $\|u\| \leq \frac{T}{\theta_{k}} \gamma_{k}(u)=\frac{T}{\theta_{k}} c_{k}$. Therefore we get

$$
0 \leq u(t) \leq \frac{T}{\gamma_{k}} c_{k}, \quad t \in[0, T]_{\mathbb{T}} .
$$

Then assumption (H5) implies

$$
f(u)<\phi\left(\frac{c_{k}}{\rho_{k}}\right), \quad t \in[0, T]_{\mathbb{T}} .
$$


So

$$
\begin{aligned}
& \gamma_{k}(T u)=\max _{t \in\left[\gamma_{k}, r_{k}\right]_{\mathbb{T}}}(T u)(t)=(T u)\left(r_{k}\right) \\
& =\int_{0}^{r_{k}} \phi^{-1}\left(\int_{s}^{T} a(r) f(u(r)) \nabla r\right. \\
& \left.-\int_{0}^{T} a(r) f(u(r)) \nabla r\right) \Delta s \\
& +\frac{\sum_{i=1}^{m-2} b_{i} \int_{0}^{\xi_{i}} \phi^{-1}\left(\int_{s}^{T} a(r) f(u(r)) \nabla r\right.}{1-\sum_{i=1}^{m-2} b_{i}} \\
& -\frac{\left.\int_{0}^{T} a(r) f(u(r)) \nabla r\right) \Delta s}{1-\sum_{i=1}^{m-2} b_{i}} \\
& -\frac{\int_{0}^{T} \phi^{-1}\left(\int_{s}^{T} a(r) f(u(r)) \nabla r\right.}{1-\sum_{i=1}^{m-2} b_{i}} \\
& -\frac{\left.\int_{0}^{T} a(r) f(u(r)) \nabla r\right) \Delta s}{1-\sum_{i=1}^{m-2} b_{i}} \\
& \leq \int_{0}^{T} \phi^{-1}\left(\int_{0}^{T} a(r) f(u(r)) \nabla r\right) \Delta s \\
& +\frac{\sum_{i=1}^{m-2} b_{i} \int_{0}^{\xi_{i}} \phi^{-1}\left(\int_{0}^{T} a(r) f(u(r)) \nabla r\right) \Delta s}{1-\sum_{i=1}^{m-2} b_{i}} \\
& -\frac{\int_{0}^{T} \phi^{-1}\left(\int_{s}^{T} a(r) f(u(r)) \nabla r\right) \Delta s}{1-\sum_{i=1}^{m-2} b_{i}} \\
& <\frac{c_{k}}{\rho_{k}}\left[\int_{0}^{T} \phi^{-1}\left(\int_{0}^{T} a(r) \nabla r\right) \Delta s\right. \\
& +\frac{\sum_{i=1}^{m-2} b_{i} \int_{0}^{\xi_{i}} \phi^{-1}\left(\int_{0}^{T} a(r) \nabla r\right) \Delta s}{1-\sum_{i=1}^{m-2} b_{i}} \\
& \left.-\frac{\int_{0}^{T} \phi^{-1}\left(\int_{s}^{T} a(r) \nabla r\right) \Delta s}{1-\sum_{i=1}^{m-2} b_{i}}\right] \\
& =c_{k} \text {. }
\end{aligned}
$$

Consequently, condition (i) is satisfied.

Secondly, we show that (ii) of Theorem 2.2 is fulled. For this we choose $u \in \partial P\left(\beta_{k}, b_{k}\right)$. Then $\beta_{k}(u)=\min _{t \in\left[r_{k}, T-\theta_{k}\right]_{\mathbb{T}}} u(t)=u\left(r_{k}\right)=b_{k}$, this means $u(t) \geq b_{k}, \quad$ for $t \in\left[r_{k}, T-\theta_{k}\right]_{\mathbb{T}}$. Therefore we have $\|u\| \geq b_{k}, \quad$ for $t \in\left[r_{k}, T-\theta_{k}\right]_{\mathbb{T}}$. Noticing that $\|u\| \leq \frac{T}{\theta_{k}} \gamma_{k}(u) \leq \frac{T}{\theta_{k}} \beta_{k}(u)=\frac{T}{\theta_{k}} b_{k}$, we get

$$
b_{k} \leq u(t) \leq \frac{T}{\theta_{k}} b_{k} \text { for } t \in\left[r_{k}, T-\theta_{k}\right]_{\mathbb{T}} .
$$

By (H6) we get

$$
f(u)>\phi\left(\frac{b_{k}}{\eta_{k}}\right), \text { for } t \in\left[r_{k}, T-\theta_{k}\right]_{\mathbb{T}} .
$$

So

$$
\begin{aligned}
& \beta_{k}(T u)=\min _{t \in\left[r_{k}, T-\theta_{k}\right]_{\mathbb{T}}}(T u)(t)=(T u)\left(r_{k}\right) \\
& =\int_{0}^{r_{k}} \phi^{-1}\left(\int_{s}^{T} a(r) f(u(r)) \nabla r\right. \\
& \left.-\int_{0}^{T} a(r) f(u(r)) \nabla r\right) \Delta s \\
& +\frac{\sum_{i=1}^{m-2} b_{i} \int_{0}^{\xi_{i}} \phi^{-1}\left(\int_{s}^{T} a(r) f(u(r)) \nabla r\right.}{1-\sum_{i=1}^{m-2} b_{i}} \\
& -\frac{\left.\int_{0}^{T} a(r) f(u(r)) \nabla r\right) \Delta s}{1-\sum_{i=1}^{m-2} b_{i}} \\
& -\frac{\int_{0}^{T} \phi^{-1}\left(\int_{s}^{T} a(r) f(u(r)) \nabla r\right.}{1-\sum_{i=1}^{m-2} b_{i}} \\
& -\frac{\left.\int_{0}^{T} a(r) f(u(r)) \nabla r\right) \Delta s}{1-\sum_{i=1}^{m-2} b_{i}} \\
& >\int_{\theta_{k}}^{r_{k}} \phi^{-1}\left(\int_{s}^{T-\theta_{k}} a(r) f(u(r)) \nabla r\right. \\
& \left.-\int_{0}^{T-\theta_{k}} a(r) f(u(r)) \nabla r\right) \Delta s \\
& \geq \frac{b_{k}}{\eta_{k}} \int_{\theta_{k}}^{r_{k}} \phi^{-1}\left(\int_{s}^{T-\theta_{k}} a(r) \nabla r\right. \\
& \left.-\int_{0}^{T-\theta_{k}} a(r) \nabla r\right) \Delta s \\
& =b_{k} \text {. }
\end{aligned}
$$

Thus, condition (ii) is satisfied.

Lastly we verify that (iii) of Theorem (2.2) is also satisfied. We note that $u(t) \equiv \frac{a_{k}}{4}, 0 \leq t \leq T$ is a member of $P\left(\alpha_{k}, a_{k}\right)$ and $\quad \alpha_{k}(u)=\frac{a_{k}}{4}<a_{k}$. Therefore $P\left(\alpha_{k}, a_{k}\right) \neq 0$. Now let $u \in \partial P\left(\alpha_{k}, a_{k}\right)$. Then $\alpha_{k}(u)=\max _{t \in\left[\theta_{k}, T-\theta_{k}\right]_{\mathbb{T}}} u(t)=u\left(T-\theta_{k}\right)=a_{k}$. This implies that $0 \leq u(t) \leq a_{k} \quad$ for $\quad t \in\left[\theta_{k}, T-\theta_{k}\right]_{\mathbb{T}}$. 
Together with $\|u\| \leq \frac{T}{\theta_{k}} \gamma_{k}(u) \leq \frac{T}{\theta_{k}} \alpha_{k}(u)=\frac{T}{\theta_{k}} a_{k}$. Then we have

$$
0 \leq u(t) \leq \frac{T}{\theta_{k}} a_{k}, \quad t \in[0, T]_{\mathbb{T}} .
$$

By (H7) we have

$$
f(u)<\phi\left(\frac{a_{k}}{\rho_{k}}\right), \quad t \in[0, T]_{\mathbb{T}} .
$$

Therefore

$$
\begin{aligned}
& \alpha_{k}(T u)=\max _{t \in\left[\theta_{k}, T-\theta_{k}\right]_{\mathbb{T}}}(T u)(t)=(T u)\left(T-\theta_{k}\right) \\
& =\int_{0}^{T-\theta_{k}} \phi^{-1}\left(\int_{s}^{T} a(r) f(u(r)) \nabla r\right. \\
& \left.-\int_{0}^{T} a(r) f(u(r)) \nabla r\right) \Delta s \\
& +\frac{\sum_{i=1}^{m-2} b_{i} \int_{0}^{\xi_{i}} \phi^{-1}\left(\int_{s}^{T} a(r) f(u(r)) \nabla r\right.}{1-\sum_{i=1}^{m-2} b_{i}} \\
& -\frac{\left.\int_{0}^{T} a(r) f(u(r)) \nabla r\right) \Delta s}{1-\sum_{i=1}^{m-2} b_{i}} \\
& -\frac{\int_{0}^{T} \phi^{-1}\left(\int_{s}^{T} a(r) f(u(r)) \nabla r\right.}{1-\sum_{i=1}^{m-2} b_{i}} \\
& -\frac{\left.\int_{0}^{T} a(r) f(u(r)) \nabla r\right) \Delta s}{1-\sum_{i=1}^{m-2} b_{i}} \\
& \leq \int_{0}^{T} \phi^{-1}\left(\int_{0}^{T} a(r) f(u(r)) \nabla r\right) \Delta s \\
& +\frac{\sum_{i=1}^{m-2} b_{i} \int_{0}^{\xi_{i}} \phi^{-1}\left(\int_{0}^{T} a(r) f(u(r)) \nabla r\right) \Delta s}{1-\sum_{i=1}^{m-2} b_{i}} \\
& -\frac{\int_{0}^{T} \phi^{-1}\left(\int_{s}^{T} a(r) f(u(r)) \nabla r\right) \Delta s}{1-\sum_{i=1}^{m-2} b_{i}} \\
& <\frac{a_{k}}{\rho_{k}}\left[\int_{0}^{T} \phi^{-1}\left(\int_{0}^{T} a(r) \nabla r\right) \Delta s\right. \\
& +\frac{\sum_{i=1}^{m-2} b_{i} \int_{0}^{\xi_{i}} \phi^{-1}\left(\int_{0}^{T} a(r) \nabla r\right) \Delta s}{1-\sum_{i=1}^{m-2} b_{i}} \\
& \left.-\frac{\int_{0}^{T} \phi^{-1}\left(\int_{s}^{T} a(r) \nabla r\right) \Delta s}{1-\sum_{i=1}^{m-2} b_{i}}\right]
\end{aligned}
$$$$
=a_{k} .
$$

Hence condition (iii) of Theorem 2.2 is satisfied. Because all hypothesses of Theorem 2.2 are satisfied, claim follows.

If we add the condition of $a(t) f(t, 0,0) \not \equiv 0, \quad t \in[0, T]_{\mathbb{T}}$, to Theorem 3.2 we can get three infinite families of positive solutions $\left\{u_{1 k}\right\}_{k=1}^{\infty}, \quad\left\{u_{2 k}\right\}_{k=1}^{\infty}$ and $\left\{u_{3 k}\right\}_{k=1}^{\infty}$ satisfying

$$
\begin{gathered}
0<\alpha_{k}\left(u_{1 k}\right)<a_{k}<\alpha_{k}\left(u_{2 k}\right), \\
\beta_{k}\left(u_{2 k}\right)<b_{k}<\beta_{k}\left(u_{3 k}\right), \\
\gamma\left(u_{3 k}\right)<c_{k}, \text { for } k \in N .
\end{gathered}
$$

\section{Example}

There exists the function $a(t)$ satisfying condition (H2). Let $\mathbb{T} \equiv 1$ and $\delta=\sqrt{2}\left(\pi^{2} / 3-9 / 4\right)$,

$$
t_{0}=\frac{5}{16}, \quad t_{n}=t_{0}-\sum_{i=0}^{n-1} \frac{1}{(i+2)^{4}}, \quad n=1,2, \ldots
$$

We consider the function $a(t):[0,1] \rightarrow(0,+\infty)$ is given by $a(t)=\sum_{n=1}^{\infty} a_{n}(t), \quad t \in[0,1]$, where

$$
a_{n}(t)= \begin{cases}\frac{2}{n(n+1)\left(t_{n+1}+t_{n}\right)}, & 0 \leq t<\frac{t_{n+1}+t_{n}}{2}, \\ \frac{1}{\delta\left(t_{n}-t\right)^{1 / 2}}, & \frac{t_{n+1}+t_{n}}{2} \leq t<t_{n} \\ \frac{1}{\delta\left(t-t_{n}\right)^{1 / 2}}, & t_{n}<t \leq \frac{t_{n}+t_{n-1}}{2} \\ \frac{2}{n(n+1)\left(2-t_{n}-t_{n-1}\right)}, & \frac{t_{n}+t_{n-1}}{2}<t \leq 1\end{cases}
$$

At first, it is easily seen that $t_{1}=1 / 4<1 / 2, t_{n}-t_{n+1}=$ $\frac{1}{(n+2)^{4}}, n=1,2, \ldots$ and (note that $\sum_{i=1}^{\infty} \frac{1}{n^{4}}=\frac{\pi^{4}}{90}$ ),

$$
\begin{aligned}
t^{*} & =\lim _{n \rightarrow \infty} t_{n}=\frac{5}{16}-\sum_{i=0}^{\infty} \frac{1}{(i+2)^{4}} \\
& =\frac{5}{16}-\left(\frac{\pi^{4}}{90}-1\right)=\frac{21}{16}-\frac{\pi^{4}}{90}>\frac{1}{5} .
\end{aligned}
$$


Next, since $\sum_{i=1}^{\infty} \frac{1}{n^{2}}=\frac{\pi^{2}}{6}$, we have

$$
\begin{aligned}
\sum_{n=1}^{\infty} \int_{0}^{1} a_{n}(t) \nabla t= & \sum_{n=1}^{\infty} \frac{2}{n(n+1)} \\
& +\frac{1}{\delta} \sum_{n=1}^{\infty}\left[\int_{\left(t_{n+1}+t_{n}\right) / 2}^{t_{n}} \frac{1}{\left(t_{n}-t\right)^{1 / 2}} \nabla t\right. \\
& \left.+\int_{t_{n}}^{\left(t_{n}+t_{n-1}\right) / 2} \frac{1}{\left(t-t_{n}\right)^{1 / 2}} \nabla t\right] \\
= & 2+\frac{\sqrt{2}}{\delta} \sum_{n=1}^{\infty}\left[\left(t_{n}-t_{n+1}\right)^{1 / 2}\right. \\
& \left.+\left(t_{n-1}-t_{n}\right)^{1 / 2}\right] \\
= & 2+\frac{\sqrt{2}}{\delta} \sum_{n=1}^{\infty}\left[\frac{1}{(n+2)^{2}}+\frac{1}{(n+1)^{2}}\right] \\
= & 2+\frac{\sqrt{2}}{\delta}\left[\left(\frac{\pi^{2}}{6}-\frac{5}{4}\right)+\left(\frac{\pi^{2}}{6}-1\right)\right] \\
= & 2+\frac{\sqrt{2}}{\delta}\left[\frac{\pi^{2}}{3}-\frac{9}{4}\right]=3 .
\end{aligned}
$$

Hence,

$$
\int_{0}^{1} a(t) \nabla t=\int_{0}^{1} \sum_{n=1}^{\infty} a_{n}(t) \nabla t=\sum_{n=1}^{\infty} \int_{0}^{1} a_{n}(t) \nabla t<\infty .
$$

This implies that condition (H2) holds.

\section{Acknowledgement}

The author would like to thank the editor and the anoymous referee for their helpful comments and suggestions.

The project is supported by Abdullah Gul University Foundation of Turkey(Project No. 5).

\section{References}

[1] D.R. Anderson, Existence of solutions for nonlinear multipoint problems on time scales, Dynamic Systems and Applications, 15, 21-34 (2006).

[2] M. Bohner, A. Peterson, Dynamic Equations on Time Scales: An Introduction with Applications, Birkhauser, Boston, Cambridge, MA, (2001).

[3] M. Bohner, A. Peterson, Advances in Dynamic Equations on Time Scales, Birkhauser, Boston, Cambridge, MA, (2003).

[4] K. Deimling, Nonlinear Functional Analysis, SpringerVerlag, New York, (1985).

[5] A. Dogan, J.R. Graef, L. Kong, Higher order semipositone multi-point boundary value problems on time scales, Computers and Mathematics with Applications, 60, 23-35 (2010).
[6] A. Dogan, J.R. Graef, L. Kong, Higher order singular multipoint boundary value problems on time scales, Proceedings of the Edinburgh Mathematical Society, 54, 345-361 (2011).

[7] A. Dogan, Existence of three positive solutions for an m-point boundary-value problem on time scales, Electronic Journal of Differential Equations, 2013, no.149, 1-10 (2013).

[8] A. Dogan, Existence of multiple positive solutions for $p$ Laplacian multipoint boundary value problems on time scales, Advances in Difference Equations, 2013, no.238, 123 (2013).

[9] W. Han, Z. Jin, S. Kang, Existence of positive solutions of nonlinear $m$-point BVP for an increasing homeomorphism and positive homomorphism on time scales, Journal of Computational and Applied Mathematics, 233, 188-196 (2009).

[10] Z. He, Double positive solutions of three-point boundary value problems for $p$-Laplacian dynamic equations on time scales, Journal of Computational and Applied Mathematics, 182, 304-315 (2005).

[11] S. Hilger, Analysis on measure chains-a unified approach to continuous and discrete calculus, Results in Mathematics, 18, 18-56 (1990).

[12] D. Ji, Z. Bai, W. Ge, The existence of countably many positive solutions for singular multipoint boundary value problems, Nonlinear Analysis, 72, 955-964 (2010).

[13] E.R. Kaufmann, Positive solutions of a three-point boundary value problem on a time scale, Electronic Journal of Differential Equations, 82, 1-11 (2003).

[14] S. Liang, J. Zhang, Z. Wang, Existence of countably many positive solutions for $n$ th-order $m$-point boundary value problems on time scales, Electronic Journal of Differential Equations, 123, 1-13 (2008).

[15] S. Liang, J. Zhang, The existence of countably many positive solutions for nonlinear singular $m$-point boundary value problem, Journal of Computational and Applied Mathematics, 214, 78-89 (2008).

[16] S. Liang, J. Zhang, The existence of countably many positive solutions for nonlinear singular $m$-point boundary value problems on time scales, Journal of Computational and Applid Mathematics, 223, 291-303 (2009).

[17] D. Ma, Z. Du, W. Ge, Existence and iteration of monotone positive solutions for multipoint boundary value problem with $p$-Laplacian operator, Computers and Mathematics with Applications, 50, 729-739 (2005).

[18] J.L. Ren, W.G. Ge, B.X. Ren, Existence of positive solutions for quasi-linear boundary value problems, Acta Mathematicae Applicatae Sinica, 21, 353-358 (2005). (in Chinese)

[19] Y. Sang, H. Su, F. Xu, Positive solutions of nonlinear $m$-point BVP for an increasing homeomorphism and homomorphism with sign changing nonlinearity on time scales, Computers and Mathematics with Applications, 58, 216-226 (2009).

[20] Y. Sang, H. Su, Several existence theorems of nonlinear $m$-point boundary value problem for $p$-Laplacian dynamic equations on time scales, Journal of Mathematical Analysis and Applications, 340, 1012-1026 (2008).

[21] Y.H. Su, Arbitrary positive solutions to a multi-point $p$ Laplacian boundary value problem involving the derivative on time scales, Mathematical and Computer Modelling, 53, 1742-1747 (2011). 
[22] H.R. Sun, Triple positive solutions for $p$-Laplacian $m$-point boundary value problem on time scales, Computers and Mathematics with Applications, 58, 1736-1741 (2009).

[23] H.R. Sun, W.T. Li, Multiple positive solutions for $p$ Laplacian $m$-point boundary value problems on time scales, Applied Mathematics and Computation, 182, 478-491 (2006).

[24] Y. Yang, F. Meng, Positive solutions of the singular semipositone boundary value problem on time scales, Mathematical and Computer Modelling, 52, 481-489 (2010).

[25] Y. Zhu, J. Zhu, The multiple positive solutions for $p$ Laplacian multipoint BVP with sign changing nonlinearity on time scales, Journal of Mathematical Analysis and Applications, 344, 616-626 (2008).

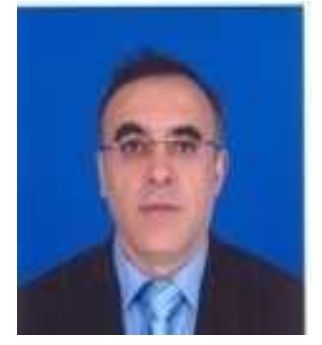

Abdulkadir Dogan received his Ph.D. in applied mathematics from University of Wales, U.K. He was a postdoctoral fellow at Leicester University, U.K. He pursued postdoctoral research at University of Tennessee at Chattanooga, USA. He joined Abdullah Gul University, Faculty of Computer Sciences in applied mathematics in December 2011. He became head of the Applied Mathematics Department in 2012. Dr Dogan's research interests include finite element methods, numerical solution of partial differential equations, boundary value problems for ordinary differential equations, and dynamic equations on time scales. He has published a lot of articles in SCI Journals and his articles have been highly cited. He gave many invited talks in various prestigious scientific meetings and academic institutions. 